\title{
A Formula For The Units To Satisfy An Operation's Desired Rate Of Return In CVP Analysis - A Conceptual Approach
}

Johann A. Anderson, CAPM, Butte County, USA

Wallace R. Leese, Ph.D., CMA, California State University, USA

\begin{abstract}
A common formula presented in many managerial-and cost-accounting textbooks makes possible the determination of the quantity of units which must be produced and sold to generate a desired dollar-amount of operating income. This article addresses the question "What formula can be used to determine the quantity of units needed to yield a desired operating-income level expressed as a rate of return on total operations-costs?" Algebraic and conceptual issues regarding the derivation of such a formula are identified. The desired formula is initially derived via a conceptual approach, which is verified with algebraic solutions afterward. An example problem is given, followed by concluding remarks.
\end{abstract}

Keywords: CVP Analysis; Rate of Return; Managerial-Accounting; Cost-Accounting

\section{INTRODUCTION}

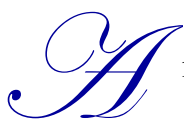
common formula ${ }^{1}$ presented in many managerial- and cost-accounting textbooks for determining the number of units which must be produced and sold to generate a desired operating income is:

\section{Equation 1}

$$
Q=\frac{T F C+O I}{C M}
$$

where the variable $Q$ represents the required number of units; the variable $T F C$, total fixed costs, represents the costs which are unchanging throughout a range of operational activity; the variable $O I$ represents operating income before related income taxes; and, finally, the variable $C M$ represents the contribution margin generated per unit sold. Equation 1 can be derived from the following formula:

\section{Equation 2}

$$
T S=T C+O I
$$

where the variable $T S$ represents total sales dollars for a given level of operational activity and the variable $T C$ represents total operations-costs for that same level of operational activity. Furthermore, total operations-costs, $T C$, can be separated in to total fixed costs, $T F C$, and total variable costs, $T V C$, for any given level of operational activity. Total variable costs are those costs which change in total in a directly proportional manner as the level of activity changes. Equation 3 expresses this cost-behavior relationship algebraically:

\footnotetext{
${ }^{1}$ For example, see: Ray H. Garrison, Eric W. Noreen, and Peter C. Brewer, Managerial Accounting 15th ed. (New York: McGraw-Hill Education, Inc., 2015), p. 203 and Charles T. Horngren, Srikant M. Datar, and Madhav V. Rajan. Cost Accounting: A Managerial Emphasis 15th ed. (Boston: Pearson Education Inc., 2015), p. 75.
} 


\section{Equation 3}

$$
T C=T V C+T F C
$$

Substituting Equation 3 into Equation 2 yields:

$$
\begin{aligned}
& T S=T V C+T F C+O I \\
& T S-T V C=T F C+O I
\end{aligned}
$$

Total sales, $T S$, minus total variable costs, $T V C$, is defined as total contribution margin, $T C M$. Therefore:

\section{Equation 4}

$$
T C M=T F C+O I
$$

Total contribution margin, $T C M$, is the product of $C M$ and $Q$, expressed formulaically as:

Equation 5

$$
T C M=Q(C M)
$$

Substituting Equation 5 into Equation 4 yields:

$$
Q(C M)=T F C+O I
$$

Dividing both sides of the above equation by $C M$ completes the derivation of Equation 1:

\section{Equation 1}

$$
Q=\frac{T F C+O I}{C M}
$$

For the solution value $Q$ to be valid, $Q$ must fall within the relevant range that is consistent with the variable values imputed into Equation 1. The relevant range is defined as that range of activity for which the assumed cost-behavior patterns hold. Thus, within the relevant range, the selling price per unit, variable cost per unit, and total fixed costs must be both known and stable. Additionally, cost-volume-profit analysis ${ }^{2}$ assumes that the number of units sold is equal to the number of units produced. This assumption is why $Q$ is defined as the number of units which must be produced and sold to generate a desired level of operating income. This assumption ensures that fixed costs are neither deferred in excess inventory (by units produced being greater than units sold in the same period) nor released from old inventory (by units sold being greater than units produced in the same period). Even when the number of units produced is equal to the number of units sold, units move in and out of inventory. Therefore, cost-volumeprofit analysis also assumes that the fixed-manufacturing-overhead rate per unit for previous manufacturing periods is the same as the fixed-manufacturing-overhead rate per unit for the current manufacturing period. This assumption ensures that total fixed costs associated with the current time period will remain stable regardless of which inventory cost-flow assumption is used. Following the same line of reasoning, unit-manufacturing variable costs for previous manufacturing periods are assumed to be the same as the unit-manufacturing variable costs for the current manufacturing period. These last two assumptions negate any possible impact caused by differences in inventory cost-flow assumptions that might recognize prior-period costs in the current period.

The Research Question: What formula can be used to determine the quantity of units needed to yield a desired operating-income level expressed as a rate of return on total operations-costs?

\footnotetext{
${ }^{2}$ Cost-volume-profit (CVP) analysis is the study of how costs and profits are affected by changes in the volume of production and sales within the relevant range of operations.
} 
Equation 1 can only be used to calculate the quantity of units, $Q$, needed to yield a desired operating-income level expressed as a dollar amount. The research issue addressed here is an expansion of Equation 1. What formula can be used to determine $Q$ when the desired operating-income level, $O I$, is expressed as a rate of return instead of as a dollar amount? The rate of return, $R$, addressed here is determined by taking a certain level of operating income, $O I$, and dividing it by the operating costs, $T C$, necessary to achieve that level of operating income. $R$ can be expressed as:

\section{Equation 6}

$$
R=\frac{O I}{T C}
$$

Note that the denominator of Equation 6 is $T C$, which can be restated as total-variable-costs and total-fixed-costs components. The rate of return determined by solving Equation 6 is not dependent on the mix of these components, only on the total costs of operations. Thus, the rate of return, $R$, earned on total variable costs is effectively the same as the rate of return earned on total fixed costs. Equation 6 defines this uniform rate of return, $R$.

\section{SOLUTION ISSUES}

From Equation 6, operating income can be defined as the product of the operating rate of return and total operating costs:

$$
O I=R(T C)
$$

To derive a formula to determine a $Q$ that will yield a desired rate of return, $R(T C)$ can be substituted for $O I$ in Equation 1, yielding:

\section{Equation 7}

$$
Q=\frac{T F C+R(T C)}{C M}
$$

The problem, now, is that the equation's right side contains the variable $T C$, whose value is dependent on the value of $Q$, which is dependent on the value of $T C$ in the current equation. Total variable costs, which are included in total operations-costs, change with the quantity of units produced and sold, meaning that one must know $Q$ to know TVC, and one must know $T V C$ to know $T C$. Therefore, one must know $Q$ to know $T C$. Thus, this formula is not useful for calculating $Q$ because the value of one of the variables, $T C$, is dependent on $Q$ first being known.

Perhaps a derivation beginning with Equation 6 will yield a better result:

\section{Equation 6}

$$
R=\frac{O I}{T C}
$$

Operating income is simply the difference between total sales dollars, $T S$, and total operations costs, $T C$. Therefore:

$$
R=\frac{T S-T C}{T C}
$$


As previously explained, total operations costs, $T C$, are the sum of total variable costs, $T V C$, and total fixed costs, TFC. Therefore:

$$
\begin{aligned}
& R=\frac{T S-(T V C+T F C)}{T V C+T F C} \\
& R=\frac{T S-T V C-T F C}{T V C+T F C}
\end{aligned}
$$

$T S$ is equal to the product of the selling price per unit, $P$, and $Q . T V C$ is equal to the product of the variable costs per unit, $V C$, and $Q$. Therefore:

\section{Equation 8}

$$
R=\frac{Q(P)-Q(V C)-T F C}{Q(V C)+T F C}
$$

Factoring $Q$ in the numerator yields:

$$
R=\frac{Q(P-V C)-T F C}{Q(V C)+T F C}
$$

The selling price per unit, $P$, minus the variable costs per unit, $V C$, is the contribution margin per unit, $C M$. Therefore:

\section{Equation 9}

$$
R=\frac{Q(C M)-T F C}{Q(V C)+T F C}
$$

At this point, there are two issues. One is algebraic, and the other is conceptual. The algebraic problem is that variable $Q$ is in both the numerator and the denominator of one side of the equation, with no obvious way to be simply isolated. With the goal being to develop an equation for $Q$, an algebraic challenge exists. This algebraic issue will be solved after identifying and solving the conceptual problem.

Conceptually, the problem is that the total-variable-costs term, $Q(V C)$, found in the denominator of Equation 9, changes as $Q$ changes while the costs of operational capacity, TFC, do not change as $Q$ changes within a given relevant range. This difference in cost behavior creates conflict by placing competing demands on the selling price, $P$. The selling price must cover costs resulting from two different types of cost behavior and generate a combined return on those costs that yields the specified rate of return for the whole operation. This conflict will be resolved shortly. First, further analysis by isolation of the cost-behavior types will reveal some useful solution insight. It will become clear that the treatment of $P$, even as a given value, is crucial to the conceptual solution.

\section{THE INSPIRATION TOWARDS A CONCEPTUAL SOLUTION}

If the total sales and total variable costs are isolated from the total fixed costs of the operation by treating total fixed costs as zero in Equation 9, it becomes clear that, for all non-zero ${ }^{3}$ values of $Q$, the rate of return, $R$, is equal to the contribution margin per unit divided by the variable costs per unit, as the $Q$ variables cancel each other in the numerator and denominator:

\footnotetext{
${ }^{3}$ In such a situation, if $Q$ is zero, there are no sales realized and no variable costs recognized (expensed), which means that the rate of return is anywhere from negative infinity to positive infinity.
} 


\section{Equation 9}

$$
\begin{aligned}
& R=\frac{Q(C M)-T F C}{Q(V C)+T F C} \\
& R=\frac{Q(C M)-0}{Q(V C)+0} \\
& R=\frac{Q(C M)}{Q(V C)} \\
& R=\frac{C M}{V C}
\end{aligned}
$$

This specific $R$ will be designated $R_{V C}$. Thus, when fixed costs are assumed to be zero and $Q$ values are non-zero:

\section{Equation 10}

$$
R_{V C}=\frac{C M}{V C}
$$

Recall that $C M$ is defined as $P$ minus $V C$. Therefore:

\section{Equation 11}

$$
R_{V C}=\frac{P-V C}{V C}
$$

Conceptually, this means that, for a given $V C$ and an unknown $P, P$ alone is the determinant variable of $R$. The variable $C M$ is only unknown because the component variable $P$ is unknown, and, thus, one can simply solve for the $P$ that will yield the desired $R$. Again, this only works for the whole operation when fixed costs are zero, and the overall objective is a formula that will work in non-zero TFC scenarios in which the value of $P$ is already given.

In a similar manner, one can isolate the total fixed costs and sales of the operation by treating variable costs as zero in Equation 8. If this is done, both $P$ and $Q$ are potentially determinant variables, as $Q$ cannot be eliminated from the equation as in the variable-cost situation:

\section{Equation 8}

$$
\begin{aligned}
& R=\frac{Q(P)-Q(V C)-T F C}{Q(V C)+T F C} \\
& R=\frac{Q(P)-Q(0)-T F C}{Q(0)+T F C}
\end{aligned}
$$

\section{Equation 12}

$$
R=\frac{Q(P)-T F C}{T F C}
$$

The first of the two variables to be given a value determines the value of the remaining variable. If $P$ is given, one can simply solve for the $Q$ that will yield the desired $R$. Again, this only works if variable costs are treated as zero. However, the overall objective is a formula that will work in non-zero $V C$ scenarios in which the value of $P$ is already given.

\section{THE CONCEPTUAL SOLUTION}

The segmentation of operation costs and related returns, as illustrated above, is what generated the idea to think of price $P$ in segments. As mentioned previously, the difference in cost behavior creates conflict by placing competing demands on the selling price, $P$. If the magnitude of $P$ is such that it only covers variable costs per unit and yields a 
return per unit that is only sufficient to yield $R$ on the variable costs, $R_{V C}$, the return on the whole operation will be insufficient to yield $R$ when fixed costs are present because, as production and sales activities increase, variable costs and total sales will increase proportionally - each additional generation of sales revenue from $P$ will be just enough to satisfy $R$ for the additional variable costs of each unit produced and sold and, therefore, will never satisfy the coverage and return needs caused by the presence of fixed costs. In such a scenario, the rate of return on the whole operation will approach, but never reach, $R$ as additional units are produced and sold. Thus, a given $P$ must be of a magnitude greater than the minimum required to yield $R$ on variable costs for $R$ to possibly be satisfied for the whole operation when fixed costs are present. Additionally, if a given $P$ is considered only for its ability to cover and yield a return on fixed costs, as in the above segmentation-illustration where fixed costs were isolated, solving for $Q$ will yield a magnitude of production and sales activity that will be insufficient to yield a return that will satisfy $R$ for the whole operation when variable costs are present. If additional units are planned to be produced and sold such that $P$ will only cover fixed costs and generate a return on those costs, the return on the whole operation will be insufficient to yield $R$ when variable costs are present because the variable costs that are necessary to support the production and sales activities will have been ignored in the calculation of $Q$.

The value of price $P$ can be viewed as something segment-able into portions - price portions whose benefits of cost coverage and return can be attributed - directed - to hypothetical cost-segments of the operation as needed. If all benefits of the possible portions of price $P$ are directed to either the variable-cost or the fixed-cost segment, one ignores the other segment's pricing need (as in the variable-cost-isolation illustration) or fails to solve for an appropriate $Q$ value that will satisfy the combined needs of both segments (as in the fixed-cost-isolation illustration). If price $P$ caters only to the coverage and return needs caused by the potential variable costs of production and sales activities, its value will likely be too low (in a normal CVP situation) to cover and yield the necessary return on fixed costs, but this is no issue if price $P$ is already given, which is assumed to be the scenario for which one is trying to develop a formula. If a given price $P$ caters only to the coverage and return needs caused by the fixed costs that are necessary for operational capacity, such activity will ignore the changes in total costs arising from the changes in variable costs that are necessary to accommodate $P$ 's catering to the coverage of and return on fixed costs.

Even though the hypothetical cost-segments of the operation (variable and fixed) need only to yield returns that will average to yield a return on the whole operation that will satisfy the desired rate of return, $R$, a given $P$ must be apportioned such that production and sales activities in each of the hypothetical cost-segments of the operation will independently - as though the other segment does not exist - yield a hypothetical return that will individually satisfy $R$ for this conceptual problem to be solved. Given a price per unit, $P$, a choice must be made - how will the portions of price be considered when solving for $Q$ ?

A given $P$ must be apportioned firstly to one hypothetical cost-segment, and the remainder must be apportioned to the other. If $P$ is to be firstly apportioned to the fixed-cost segment, another decision must be made - how will it be apportioned? Satisfaction of the coverage and return needs of the fixed-cost segment is dependent upon both the price apportioned to the segment and the value of $Q$, which means that almost any value of price apportionment is valid - any non-zero price portion for that segment will suffice because the value of $Q$ can be adjusted such that the product of the price portion and $Q$ will yield the desired rate of return on the hypothetical cost-segment. Apportioning price firstly to the fixed-cost segment seems to create only one constraint, the non-zero constraint. With only that constraint, the price apportionment to the fixed-cost segment is effectively arbitrary. An arbitrary apportionment made to the fixed-cost segment will likely cause a problem, which is similar to the one previously described when variable costs are not considered in the calculation of $Q$. Before the problem is explained, recall that, if one segment yields a rate of return equal to $R$, so must the other segment, otherwise the average rate of return for the whole operation will not be $R$.

Once the value of $Q$ is determined by a calculation based on the arbitrary apportionment of $P$ to the fixed-cost segment, the rate of return for the fixed-cost segment will be equal to $R$, but what will guarantee that the rate of return for the variable-cost segment will be equal to $R$ ? The value of $Q$ will have already been determined by the fixed-cost segment, meaning that it can't be changed to accommodate the needs of the variable-cost segment, but this is irrelevant because, as has been shown in a prior illustration, the value of $Q$ does not determine the rate of return for the variable-cost segment as long as it is a non-zero value. The price apportionment to the variable-cost 
segment is the sole determinant of the rate of return of that hypothetical cost-segment, and, if the price portion does not yield a rate of return on $V C$ that is equal to $R$, each additional unit produced and sold will bring the operating rate of return further from $R$ if the rate of return for the fixed-cost segment is already $R$.

The alternative is to firstly apportion $P$ to the variable-cost segment. This is logical because the value of $Q$ can be adjusted in the fixed-cost segment to accommodate any non-zero price apportionment to that segment, but the price apportionment to the variable-cost segment is the sole determinant of the return on that segment as long as $Q$ is a non-zero value. The value of $Q$ cannot be adjusted to accommodate a price apportionment to the variable-cost segment. Thus, a constraint exists to make the price apportionment to the variable-cost segment non-arbitrary. An apportionment of $P$ firstly to the variable-cost segment can be expressed formulaically as:

\section{Equation 13}

$$
P_{C}=P_{V C r}+P_{R e m}
$$

The variable $P_{C}$ is the complete price per product unit, and it is equal to what is normally designated as $P$ in the standard CVP equation. The variable $P_{R e m}$ is the remainder of $P_{C}$ that was not apportioned to $P_{V C r}$. The variable $P_{V C r}$ represents the portion of $P_{C}$ that covers the variable costs and yields a return on those costs. Therefore, $P_{V C r}$ can further be segmented into two portions:

\section{Equation 14}

$$
P_{V C r}=P_{p 1}+P_{p 2}
$$

The variable $P_{p 1}$ represents the portion of $P_{V C r}$ that covers variable costs, and $P_{p 2}$ represents the portion of $P_{V C r}$ that yields a return on the variable costs. Because the price necessary to cover all variable costs and yield a particular rate of return on those total variable costs is effectively not dependent upon the value of $Q^{4}$, one can equate each of $P_{p 1}$ and $P_{p 2}$ to $V C$ and $V C(R)$, respectively. The result is:

\section{Equation 15}

$$
P_{p 1}+P_{p 2}=V C+V C(R)
$$

This allows the creation of an apportioned price that will continue to contribute the same desired rate of return for all non-zero values of $Q$ for this hypothetical variable-cost-segment of the operation independently of the activities in the hypothetical fixed-cost-segment of the operation.

As previously mentioned, the variable $P_{R e m}$ is the remainder of $P_{C}$ that was not apportioned to $P_{V C r}$. Because the remainder of $P_{C}$ that was not apportioned to $P_{V C r}$ must cover $T F C$ and yield a return on TFC (if possible), that remainder must be equal to a functionally similar counterpart of $P_{V C r}$, which will be designated as $P_{F C r}$. Thus:

\section{Equation 16}

$$
P_{\text {Rem }}=P_{F C r}
$$

Recall that, in Equation 13:

$$
P_{C}=P_{V C r}+P_{\text {Rem }}
$$

\footnotetext{
${ }^{4}$ Recall that, if $Q$ is zero, no price per unit sold can possibly generate a return on variable costs that would yield a specific rate of return - the implications of $Q$ are irrelevant, even when it is zero.
} 
With $P_{F C r}$ substituted for $P_{R e m}$ from Equation 16:

$$
\begin{aligned}
& P_{C}=P_{V C r}+P_{F C r} \\
& P_{V C r}+P_{F C r}=P_{C} \\
& P_{F C r}=P_{C}-P_{V C r}
\end{aligned}
$$

With $\left(P_{p 1}+P_{p 2}\right)$ substituted for $P_{V C r}$ from Equation 14:

$$
P_{F C r}=P_{C}-\left(P_{p 1}+P_{p 2}\right)
$$

With $(V C+V C(R))$ substituted for $\left(P_{p 1}+P_{p 2}\right)$ from Equation 15:

$$
P_{F C r}=P_{C}-(V C+V C(R))
$$

\section{Equation 17}

$$
P_{F C r}=P_{C}-V C(1+R)
$$

Equation 17 will be important for a later substitution.

By making $P_{F C r}$ dependent on $P_{V C r}$, one allows the proper apportionment of $P_{C}$ first to $P_{V C r}$ that will not later conflict $^{5}$ with one's effort to solve for $Q$. As was mentioned, $P_{F C r}$ is the functionally similar counterpart of $P_{V C r}$, but its ability to yield an appropriate return is dependent upon $Q$ because the related costs represented by $T F C$ are not dependent upon $Q$ as the costs represented by $T V C$ are dependent. Like $P_{V C r}, P_{F C r}$ can further be segmented into two portions, $P_{p 3}$ and $P_{p 4}$ :

\section{Equation 18}

$$
P_{F C r}=P_{p 3}+P_{p 4}
$$

The variable $P_{p 3}$ represents the portion of $P_{F C r}$ that, for a certain value of $Q$, covers fixed costs, and $P_{p 4}$ represents the portion of $P_{F C r}$ that, for a certain value of $Q$, yields a return on those fixed costs. Because one has already ensured that the rate of return on variable costs will be equal to $R$ (recall Equations 14 and 15), the rate of return on fixed costs must also be equal to $R$, otherwise the returns from both segments will not total to be the appropriate return on the operation that is necessary to yield $R$. Therefore:

\section{Equation 19}

$$
Q\left(P_{p 3}\right)=T F C
$$

\section{Equation 20}

$$
Q\left(P_{p 4}\right)=T F C(R)
$$

\footnotetext{
${ }^{5}$ Arbitrarily apportioning some of $P_{C}$ first to $P_{F C r}$ and solving for $Q$ based on that apportionment can create conflict with $P_{V C r}$ because apportioning the remainder of $P_{C}$ to $P_{V C r}$ can yield a rate of return on $V C$ that is different from $R$ and, therefore, cause each unit of activity in $Q$ to bring the actual operating rate of return further from $R$. By making the determination of $P_{F C r}$ non-arbitrary, one allows a solution for $Q$ to be achievable by one formula.
} 
Now, with the relevance and presence of $Q$ established, one can begin to solve for a $Q$ value that will satisfy the needs of $P_{F C r}$ by summing Equations 19 and 20:

$$
\begin{aligned}
& Q\left(P_{p 3}\right)+Q\left(P_{p 4}\right)=T F C+T F C(R) \\
& Q\left(P_{p 3}+P_{p 4}\right)=T F C+\operatorname{TFC}(R)
\end{aligned}
$$

Equation 18 established that:

$$
P_{F C r}=P_{p 3}+P_{p 4}
$$

With $P_{F C r}$ substituted for $\left(P_{p 3}+P_{p 4}\right)$ :

\section{Equation 21}

$$
\begin{aligned}
& Q\left(P_{F C r}\right)=T F C+T F C(R) \\
& Q\left(P_{F C r}\right)=T F C(1+R) \\
& Q=\frac{T F C(1+R)}{\left(P_{F C r}\right)}
\end{aligned}
$$

And, after making the substitution that was previously foretold from Equation 17, one has the desired formula with an additional substitution of $P$ for $P_{C}$ :

\section{Equation 17}

$$
P_{F C r}=P_{C}-V C(1+R)
$$

\section{Equation 22}

$$
Q=\frac{T F C(1+R)}{(P-V C(1+R))}
$$

\section{THE ALGEBRAIC SOLUTIONS}

A strictly algebraic approach can be used to verify the validity of the conceptually derived Equation 22 . Two algebraic derivations will be demonstrated.

The first algebraic solution starts with Equation 2:

\section{Equation 2}

$$
T S=T C+O I
$$

Equation 6 can be solved for $O I$ as follows:

\section{Equation 6}

$$
\begin{aligned}
& R=\frac{O I}{T C} \\
& O I=R(T C)
\end{aligned}
$$


Now, $R(T C)$ can be substituted for $O I$ in Equation 2 to yield:

$$
\begin{aligned}
& T S=T C+R(T C) \\
& T S=T C(1+R)
\end{aligned}
$$

Equation 3 allows the substitution of $(T V C+T F C)$ for $T C$ :

\section{Equation 3}

$$
\begin{aligned}
& T C=T V C+T F C \\
& T S=(T V C+T F C)(1+R)
\end{aligned}
$$

\section{Equation 23 ${ }^{\mathrm{i}}$}

$$
\begin{aligned}
& T S=T V C+T V C(R)+T F C+T F C(R) \\
& T S=T V C(1+R)+T F C(1+R)
\end{aligned}
$$

Recall that $T S$ is the product of variables $Q$ and $P$, and that $T V C$ is the product of variables $Q$ and $V C$. With these substitutions, the conceptually-derived Equation 22 can be algebraically derived as follows:

$$
\begin{aligned}
& Q(P)=Q(V C)(1+R)+\operatorname{TFC}(1+R) \\
& Q(P)-Q(V C)(1+R)=\operatorname{TFC}(1+R) \\
& Q(P-V C(1+R))=\operatorname{TFC}(1+R)
\end{aligned}
$$

\section{Equation 22}

$$
Q=\frac{T F C(1+R)}{(P-V C(1+R))}
$$

Equation 22 can also be derived algebraically by starting from Equation 9, the point at which the algebraic and conceptual issues were recognized:

\section{Equation 9}

$$
\begin{aligned}
& R=\frac{Q(C M)-T F C}{Q(V C)+T F C} \\
& R(Q(V C)+T F C)=Q(C M)-T F C \\
& R(Q)(V C)+R(T F C)=Q(C M)-T F C \\
& R(Q)(V C)-Q(C M)=-T F C-R(T F C)
\end{aligned}
$$

Multiply both sides of the equation by negative one:

$$
\begin{aligned}
& Q(C M)-R(Q)(V C)=T F C+R(T F C) \\
& Q(C M-R(V C))=T F C+R(T F C) \\
& Q(C M-R(V C))=T F C(1+R)
\end{aligned}
$$


Replace $C M$ with $(P-V C)$ :

$$
\begin{aligned}
& Q(P-V C-R(V C))=T F C(1+R) \\
& Q(P-(V C+R(V C)))=T F C(1+R) \\
& Q(P-V C(1+R))=T F C(1+R)
\end{aligned}
$$

\section{Equation 22}

$$
Q=\frac{T F C(1+R)}{(P-V C(1+R))}
$$

\section{DEMONSTRATION PROBLEM}

A person wants to achieve a minimum $15 \%$ return on the total costs of an operation where total fixed costs are estimated to be $\$ 14,000$, variable costs are estimated to be $\$ 12$ per unit manufactured and sold, and the sale price per unit is anticipated to be $\$ 23$. This information can be summarized as:

$$
\begin{aligned}
& R=0.15 \\
& T F C=\$ 14,000 \\
& V C=\$ 12 \\
& P=\$ 23
\end{aligned}
$$

Using Equation 22, the quantity of units that must be produced and sold to achieve the desired operating rate of return can be determined as follows:

\section{Equation 22}

$$
\begin{aligned}
& Q=\frac{T F C(1+R)}{(P-V C(1+R))} \\
& Q=\frac{14,000(1+0.15)}{(23-12(1+0.15))} \\
& Q=\frac{14,000(1.15)}{(23-12(1.15))} \\
& Q=\frac{16,100}{(23-13.8)} \\
& Q=\frac{16,100}{9.2} \\
& Q=1,750
\end{aligned}
$$

According to the solution above, 1,750 units must be produced and sold to achieve a $15 \%$ return on this operation. This can be confirmed with Equation 8: 


\section{Equation 8}

$$
\begin{aligned}
& R=\frac{Q(P)-Q(V C)-T F C}{Q(V C)+T F C} \\
& R=\frac{1,750(23)-1,750(12)-14,000}{1,750(12)+14,000} \\
& R=\frac{40,250-21,000-14,000}{21,000+14,000} \\
& R=\frac{40,250-35,000}{35,000} \\
& R=\frac{5,250}{35,000} \\
& R=0.15
\end{aligned}
$$

\section{CONCLUSION}

A common formula presented in many managerial- and cost-accounting textbooks makes possible the determination of the quantity of units which must be produced and sold to generate a desired dollar-amount of operating income (Equation 1). The research issue addressed in this thesis, "What formula can be used to determine the quantity of units needed to yield a desired operating-income level expressed as a rate of return on total operations-costs?," was solved conceptually and algebraically to yield the following formula:

$$
Q=\frac{T F C(1+R)}{(P-V C(1+R))}
$$

This formula is constrained by the usual assumptions associated with cost-volume-profit analysis. The concept of a relevant range is critical to understanding cost-volume-profit analysis. The relevant range is defined as that range of activity for which the assumed cost-behavior patterns hold. Thus, within the relevant range, the selling price per unit, variable cost per unit, and total fixed costs must be both known and stable. The formula for $Q$ may yield a solution value that is outside of the relevant range. Such a non-feasible solution would require the user to re-solve the equation for $Q$ using a new set of behavioral assumptions defining a new relevant range. Thus, an iterative solution process might be necessary to ensure a feasible solution.

Note that the formula can be used to solve for all solvable values of $Q$. When $P$ is equal to $P_{V C r}$, the denominator will be zero, representing the fact that there is no price remainder to cover and yield a return on fixed costs, meaning that no value of $Q$ can possibly cover fixed costs and yield the necessary return on those costs, and, therefore, the unit price is insufficient to achieve the desired rate of return on the whole operation (if fixed costs are present). Thus, the value of $Q$ can range from negative infinity to positive infinity in that situation, as represented by the division of a zero-value denominator into the numerator (there is no price remainder to determine the direction of $Q$ - zero is neither negative nor positive, thus, infinite values in a negative or positive direction are equally valid). Also note that, if fixed costs are not present, $Q$ is irrelevant because the rate of return on variable costs, as previously explained, is not dependent on $Q$ as long as $Q$ is a non-zero value (no specific value of $Q$ is needed - any non-zero value of $Q$ will suffice). Thus, the numerator becomes zero and the answer for $Q$ becomes zero, representing its irrelevance in that situation.

\section{AUTHOR BIOGRAPHIES}

Johann A. Anderson is a former accounting student of Dr. Wallace R. Leese (Cost Accounting, Advanced Cost Management Systems). He earned a B.S. degree in Business Administration - Accounting from California State University, Chico in May 2014, the semester in which the article was started. He was granted certification as a Certified Associate in Project Management (CAPM) in June 2013. He has worked as a staff accountant at Digital 
Path, Inc., currently works as an accountant for Butte County, and plans to return to school for an engineering degree.

Wallace R. Leese earned a BBA degree (Accounting) from Niagara University (1969), a MSA degree (Accounting) (1970) and a Ph.D. (Accounting) (1978) from Arizona State University. He is presently a Professor of Accounting at California State University, Chico. His teaching interests include principles, managerial, intermediate, cost, and advanced cost. He is a CMA. Professor Leese has served CSUC as Chairman of the Department of Accounting \& Management Information Systems, as Director of Graduate Business Programs, and as Associate Dean of the College of Business. Before entering academia, he worked as an auditor for Haskins \& Sells. 


\section{ENDNOTES}

${ }^{i}$ Endnote 1: Equation 23 can be derived from other statements and derivation steps in this article. First, recall Equations 14 and 15:

\section{Equation 14}

$$
P_{V C r}=P_{p 1}+P_{p 2}
$$

\section{Equation 15}

$$
P_{p 1}+P_{p 2}=V C+V C(R)
$$

Things that are equal to a common thing are also equal to each other. Therefore:

$$
P_{V C r}=V C+V C(R)
$$

Multiplying both sides of the equation by $Q$ yields:

$$
Q\left(P_{V C r}\right)=Q(V C)+Q(V C)(R)
$$

The product of $Q$ and $V C$ is $T V C$. Substitution yields:

\section{Equation 24}

$$
Q\left(P_{V C r}\right)=T V C+T V C(R)
$$

Note the similarities between the right side of Equation 23 and the right sides of Equations 21 and 24:

\section{Equation 23}

$$
T S=T V C+T V C(R)+T F C+T F C(R)
$$

\section{Equation 24}

$$
Q\left(P_{V C r}\right)=T V C+T V C(R)
$$

Equation 21

$$
Q\left(P_{F C r}\right)=T F C+T F C(R)
$$

Summing the previous two equations yields:

$$
Q\left(P_{V C r}\right)+Q\left(P_{F C r}\right)=T V C+T V C(R)+T F C+T F C(R)
$$

Factoring $Q$ yields:

$$
Q\left(P_{V C r}+P_{F C r}\right)=T V C+T V C(R)+T F C+T F C(R)
$$

Recall that $P_{C}$ equals the sum of $P_{V C r}$ and $P_{F C r}$, which was established during the reasoning for Equation 16 and shown formulaically afterward when deriving Equation 17. Therefore:

$$
Q\left(P_{C}\right)=T V C+T V C(R)+T F C+T F C(R)
$$

The variable $P_{C}$ is merely another expression of price $P$, and, thus, Equation 23 represents the fact that the product of the selling price per unit and the quantity of units must cover total costs resulting from two types of cost behavior and yield returns on those costs that satisfy $R$. 